\title{
Classification of Power Quality Disturbances in Electric Power System: A Review
}

\author{
Devendra Mittal ${ }^{1}$, Om Prakash Mahela ${ }^{2}$, Rohit Jain ${ }^{3}$ \\ ${ }^{1}$ (Assistant Professor, Department of Electrical Engineering, Jagannath University Jaipur, India) \\ ${ }^{2}$ (Junior Engineer, Rajasthan Rajya Vidhyut Prasaran Nigam Ltd., Jaipur, India) \\ ${ }_{3}^{3}$ (Professor, Department of Physics, Jagannath Gupta Institute of Engineering \& Technology, Jaipur, India)
}

\begin{abstract}
The Electrical power quality issue has attained considerable attention in last decade due to large penetration of power electronics based loads and microprocessor based controlled loads. These devices introduce power quality problem on one hand and these mal-operate due to induced power quality problems on other hand. The various techniques for classification or recognition of power quality disturbances in electrical power system have been proposed so far in different papers. This paper presents a comprehensive overview of different techniques used for feature extraction and classification of power disturbance. This paper helps the researchers to know about the different methods presented so far for power quality disturbance classification or recognition, so that further work on power quality improvement can be carried out for better results.
\end{abstract}

Keywords: artificial intelligence techniques, feature extraction, genetic algorithm, $P Q$ disturbances, power system, power quality, $P Q$ event classifier.

\section{Introduction}

Electrical power system is expected to deliver undistorted sinusoidal rated voltage and current continuously at rated frequency to the consumers. In recent years, grid users have detected an increasing number of drawbacks caused by electric power quality (PQ) variations and PQ problems have sharpened because of the increased number of loads sensitive to PQ and have become more difficult to solve as the loads themselves have become important causes of degradation of quality [1].Therefore, these days, customers demand higher levels of PQ to ensure the proper and continued operation of such sensitive equipments. According to IEEE standard 1159-1995 [2], the PQ disturbances include wide range of PQ phenomena namely transient (impulsive and oscillatory), short duration variations (interruption, sag and swell), power frequency variations, long duration variations (sustained under voltages and sustained over voltages) and steady state variations (harmonics, notch, flicker etc.) with time scale ranges from tens of nanoseconds to steady sate. A number of causes of transients can be identified: lightning strokes, planned switching actions in the distribution or transmission system, selfclearing faults or faults cleared by current limiting fuses, and the switching of end-user equipment. Transient phenomena are extremely critical since they can cause over voltages leading to insulation breakdown or flashover. These failures might trip any protection device initiating a short interruption to the supplied power. Excess current produced by transients may lead to complete damage to system equipment during the transient period. Moreover, if such disturbances are not mitigated, they can lead to failures or malfunctions of various sensitive loads in power systems and may be costly. According to the survey of IEEE Transactions on Industrial Applications, power quality disturbances lead to losses of $\$ 4$ billion to $\$ 10$ billion in the USA alone [3].

In electricity market scenario, now electricity consumers can shift to the new service providers, if power quality is not good. Moreover, these customers can demand a higher quality of service. The utilities or other electric power providers have to ensure a high quality of their service to remain competitive and to retain/ attract the customers. Therefore the Power Quality has been a challenge for power system planners and researchers. The main task of PQ analysis involves detection, identification, recognition and classification of various types of PQ disturbances. In this work, an analysis of PQ issues, types of PQ disturbances, automatic power quality recognition system, feature extraction techniques and artificial intelligence based classification methods proposed by the researchers recently are presented.

II. Power Quality and Types of Power Quality Disturbances

The term power quality (PQ) is generally applied to a wide variety of electromagnetic phenomena occurring within a power system network. Power quality is predominantly a customer issue. Power quality can be defined as any problem manifested in voltage, current, or frequency deviation that results in failure or maloperation of electric equipment [4]. The electric power quality is also defined as a term that refers to maintaining the near sinusoidal waveform of power system bus voltages and currents at rated magnitude and frequency. Thus electric power quality is often used to express voltage quality, current quality, reliability of 
service, quality of power supply etc. [5]. Power quality issue is also important for the utility companies. They are obliged to supply consumers with electrical power of acceptable quality.

The power quality disturbances depend on amplitude or frequency or on both frequency and amplitude. Based on duration of existence of PQ disturbances, events can be divided in to short, medium or long type. The classification and identification of each disturbance are usually carried out from standards and recommendations depending on where the utilities operate (e.g. IEEE in the U.S.). Inigo Monedero et al. [6] defined PQ disturbances, which is given in Table I, based on the UNE standard in Spain which defines the ideal signal as a single-phase or three-phase sinusoidal voltage signal of $230 \mathrm{~V}_{\mathrm{RMS}}$ and $50 \mathrm{~Hz}$. D. Saxena et al. [7] classified various PQ events in to five groups viz. short duration variation, long duration variation, transients, voltage imbalance and waveform distortion. S.Edwin Jose et al. [8] classified PQ disturbances on basis of values of tails of histogram obtained from simulation results.

TABLE I

TYPES OF DISTURBANCES

\begin{tabular}{|c|c|c|c|c|c|c|}
\hline \multirow[b]{2}{*}{$\begin{array}{c}\text { Type of } \\
\text { disturbance }\end{array}$} & \multirow{2}{*}{\multicolumn{3}{|c|}{ Disturbance subtype }} & \multirow[b]{2}{*}{$\underline{\text { Time }}$} & \multicolumn{2}{|c|}{ Range } \\
\hline & & & & & Min. Value & $\frac{\text { Max. }}{\text { Value }}$ \\
\hline \multirow{2}{*}{ Frequency } & \multicolumn{3}{|c|}{ Slight deviation } & \multirow{2}{*}{$10 \mathrm{~s}$} & $49.5 \mathrm{~Hz}$. & $50.5 \mathrm{~Hz}$. \\
\hline & \multicolumn{3}{|c|}{ Severe deviation } & & $47.0 \mathrm{~Hz}$. & $52.0 \mathrm{~Hz}$. \\
\hline \multirow{11}{*}{ Voltage } & \multicolumn{3}{|c|}{ Average voltage } & $10 \mathrm{~min}$ & $0.85 \mathrm{Un}$ & $1.1 \mathrm{Un}$ \\
\hline & \multicolumn{3}{|c|}{ Flicker } & - & - & $7 \%$ \\
\hline & \multirow{3}{*}{ Sag } & & & $10 \mathrm{~ms}-1 \mathrm{~s}$ & \multirow{3}{*}{$0.1 \mathrm{U}$} & \multirow{3}{*}{$0.9 \mathrm{U}$} \\
\hline & & & & $1 \mathrm{~s}-1 \mathrm{~min}$ & & \\
\hline & & \multicolumn{2}{|c|}{ Long-time disturbance } & $>1 \mathrm{~min}$ & & \\
\hline & \multirow{2}{*}{\multicolumn{2}{|c|}{ Under Voltage }} & Short & $<3 \min$ & \multirow{2}{*}{\multicolumn{2}{|c|}{$0.99 \mathrm{U}$}} \\
\hline & & & Long & $>3 \min$ & & \\
\hline & \multirow{4}{*}{ Swell } & \multicolumn{2}{|c|}{ Temporary Short } & $10 \mathrm{~ms}-1 \mathrm{~s}$ & \multirow{4}{*}{$1.1 \mathrm{U}$} & \\
\hline & & & ong & $1 \mathrm{~s}-1 \mathrm{~min}$ & & $1.5 \mathrm{KV}$ \\
\hline & & Ten & g-time & $>1 \mathrm{~min}$ & & \\
\hline & & & & $<10 \mathrm{~ms}$ & & $6 \mathrm{KV}$ \\
\hline \multirow{2}{*}{$\begin{array}{c}\text { Harmonics } \\
\text { and other } \\
\text { information } \\
\text { signals }\end{array}$} & \multicolumn{3}{|c|}{ Harmonics } & - & \multicolumn{2}{|c|}{ THD> $>8 \%$} \\
\hline & \multicolumn{3}{|c|}{ Information signals } & - & \multicolumn{2}{|c|}{$\begin{array}{l}\text { Included in other } \\
\text { disturbances }\end{array}$} \\
\hline
\end{tabular}

III. Automatic Power Quality Disturbance Recognition System

The PQ disturbance recognition scheme consists of three major parts viz. (i) feature extraction i.e. signal processing (ii) Classification of disturbance using conventional or artificial intelligence (AI) based techniques and (iii) the decision making as to what category a particular disturbance belongs. A scheme for automatic power quality disturbance recognition system is depicted in Fig. 1 [9].

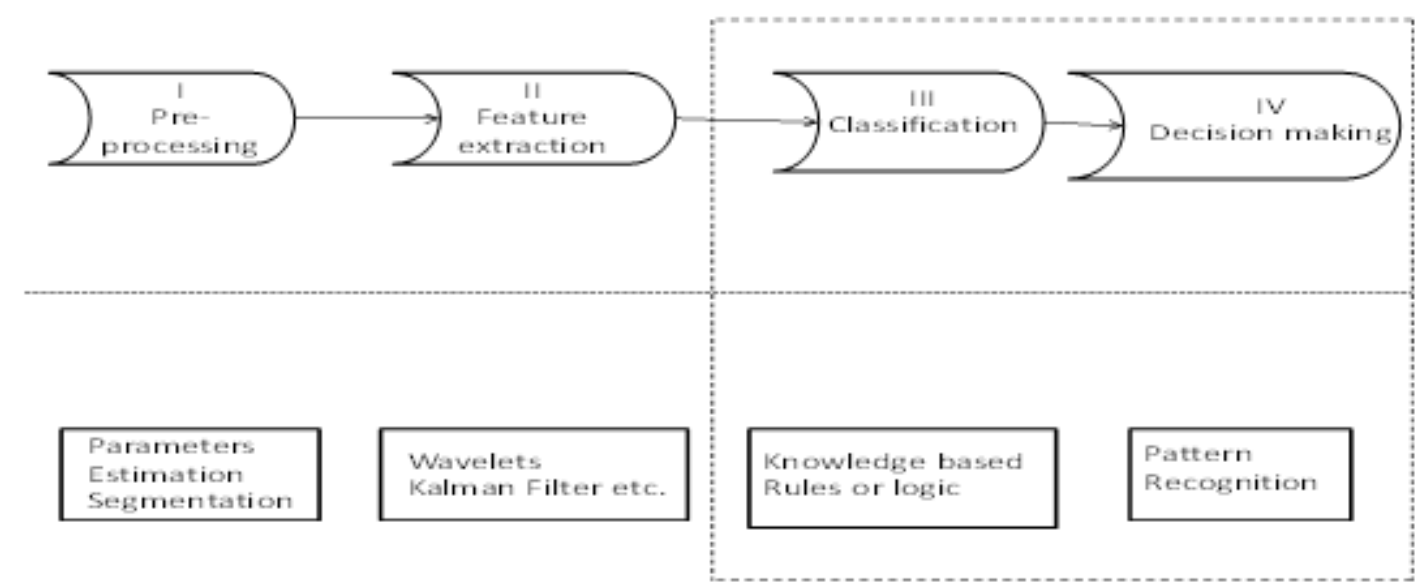

Fig. 1 block diagram of automatic disturbance recognition system 
In the figure, Block-I represents a pre-processing stage. In this block estimation of signal components is performed. Then, an algorithm for signal segmentation in different stages is applied, e.g. pre-event, duringevent and post-event stages. Block-II represents a feature extraction stage. Feature extraction can be done through any of the techniques such as Fourier transforms, S-transform, wavelet transform, Hilbert Huang transform etc. Block-III represents the classification stage based on defined rules, e.g. knowledge based expert systems, pattern recognition or any logic to discriminate different types of events. The artificial neural network, support vector machine based system, rule-based expert system, fuzzy expert system, genetic algorithm, adaptive neuro-fuzzy system etc. are commonly used for classification of PQ events. Finally, Block-IV represents the decision making stage. In this stage the type of event is assigned to an actual type event. In most of the classifiers, the decision making stage is merged with the classification stage. Normally, expert system and fuzzy logic are used as decision making tools.

\section{Feature Extraction Techniques}

In order to improve the quality of electric power supplied, it is essential to detect and identify the power quality problem distinctive features of the disturbance waveforms. Feature may directly be extracted from the original measurement (e.g. RMS values), or from some transformed domain (e.g. Fourier, wavelet, STFT, HHT, and S-transform), or from the parameters of signal models (e.g. sinusoid KF and AR models). The RMS magnitude of voltage supply is used in the power quality standards for detection and characterization of voltage events [10]. The method is simple and easy to implement but it does not give information about the phase angle or the point on wave where the event begins [5]. RMS method has important limitations in the detection and estimation of magnitude and duration of voltage events. Commonly used feature extraction techniques are mentioned below:

\section{IV.1 Fourier Transform}

The most used classical signal processing is the Fourier transform (FT). This transform represents a signal as a sum of sinusoidal terms of different frequencies, named frequency spectrum [11]. This technique is suitable for stationary signals and extracting spectrum components of the signals at specific frequencies, but it is not efficient when the signal contains short-term transient disturbances [12]. P. Kailasapathi et al. [13] described that FT can be applied to both continuous signal and discrete signal. It can be either periodic or aperiodic. Anton et al. [14] presented that the FT based signal processing techniques are Discrete Fourier Transform (DFT), Fast Fourier Transform (FFT) and Short Time Fourier Transform (STFT).

The DFT is the widely used discrete signal processing algorithm. An approach to compute the DFT is to use a recursive computation scheme. The most popular approach is the Goertzel's Algorithm. Goertzel algorithm is used to implement the non-uniform discrete fourier transform [15]. Rosendo et al. [16] described that in the windowed discrete Fourier transform (WDFT), the amplitude for any given spectral component of the DFT gives the average of windowed tie series. The choice of window size is, however, completely arbitrary.

An FFT computes the DFT and produces exactly the same result as evaluating the DFT definition directly; the only difference is that an FFT is much faster. The radix is the size of FFT decomposition. Radix-2, radix-4, radix- 8 , spilit radix are the types of radix. The FFT algorithms are found to be more accurate than evaluating the DFT definition direct computation when round-off error is present [17].

The STFT is the classical method of time frequency analysis. It is a Fourier related transform used to determine the sinusoidal frequency and phase content of local sections of a signal as it changes over time. In essence, STFT extracts several frames of the signal to be analyzed with a window that moves with time. If the time window is sufficiently narrow, each frame extracted can be viewed as stationary so that Fourier transform can be used. With the window moving along the time axis, the relation between the variance of frequency and time can be identified [18]-[20]. Non-stationary signals characterized by wide range of frequency spectrum with transient and sub-harmonic components are difficult to analyze with STFT [21]. For non-stationary signals, the STFT does not track the signal dynamics properly due to the limitations of a fixed window width chosen a priority [19].

\section{IV.2 S-Transform}

Stockwell et al. [22] introduced the S-Transform (ST) to track the dynamics properly. The S-transform is a time-frequency spectral localization method, similar to the STFT and continuous wavelets. The S-transform is conceptually a hybrid of STFT and wavelet analysis, bridges the gap between them, containing elements of both but having its own characteristic properties. Just like the STFT, the S-transform uses a window to localize the complex Fourier sinusoid, but unlike the STFT, the width and height of the window scale with frequency in analogy with wavelets. S-transform employs a moving and scalable localizing Gaussian window. It combines a frequency dependent resolution with simultaneous localizing the real and imaginary spectra [23]-[24]. Leonowicz et al. [25] stated that the basic functions for the S-transform are Gaussian modulated cosinusoids 
whose width varies inversely with the frequency. Ming He Zhang et al. [26] employed S-Transform for the analysis and detection of power quality disturbances. Ameen M. Gargoom et al. [27] proposed the analysis of power quality events using amplitude and phase spectrum derived from the S-Transform of signal. Nguyen et al. [28] presented Discrete S-Transform (DST) for feature extraction. Yokeeswaran et al. [29] presented Discrete Orthogonal S-Transform (DOST) using time series for representation. The transformation matrix is orthogonal, meaning that the inverse matrix is equal to the complex conjugate transpose. An orthogonal transform is referred to as an energy preserving transform.

\section{IV.3 Wavelet Transform}

Wavelet Transform (WT) is used as a feature extraction tool to identify power quality disturbances [30]. The wavelet transform traces the signal changes in time domain and simultaneously decomposes the signal in frequency domain. In the wavelet transform based approach, a mother wavelet is employed for finding the wavelet coefficients of the signal which affects the effectiveness in identifying the disturbance present in the signal [31]. The mathematics of wavelet transform was extensively studied and can be referred in papers [32] and [33]. Wavelet transforms are classified into discrete wavelet transform (DWT) and continuous wavelet transforms (CWT). Lalit Behera et al. [34] presented the theory of CWT and DWT and used DWT for time series data mining of power quality events occurring due to power signal disturbances. The wavelet transform has been introduced as powerful tool for voltage flicker signal extraction and harmonic detection [35]. The main disadvantage of WT is its batch processing, which in turn, results in some delay. Feature extraction technique using the standard deviation of the wavelet coefficients is discussed in [36]. Perumal et al. [37] employed multi-resolution analysis (MRA) based modified wavelet transforms for detection and classification of power quality disturbance waveform. The use of continuous wavelet transform (CWT) to analyze non-stationary harmonic distortion has been proposed in [38].

\section{IV.4 Hilbert Huang Transform}

The development of the Hilbert Huang Transform (HHT) was motivated by the need to describe nonlinear distorted waves in detail, along with the variations of these signals that naturally occur in nonstationary processes [39].The Hilbert Huang Transform is an adaptive data analysis method designed for analyzing non-stationary signals. In HHT, the signal is decomposed into a finite small number of components, called Intrinsic Mode Functions (IMF). This process of decomposition is called Empirical Mode decomposition (EMD). The EMD decomposes the signal in terms of IMFs each of which is a mono-component function. A signal can be analyzed in details for its frequency, amplitude and phase contents by using EMD followed by HT [40]. The method has been applied to many important problems in various fields including medical [41], geophysics [42] and power engineering [43]. Jayasree et al. [44] employed automated classification of power quality disturbances using HHT and RBF neural networks. Nilanjan Senroy et al. [45] presented an innovative algorithm of improved Hilbert Huang method based on masking signals for power quality applications. The rationale for the improvements is that the original EMD method is unable to separate frequency modes lying within one octave. The proposed method is shown to efficiently separate modes existing in distorted signals typically encountered in power quality applications. Elango et al. [46] presented performance comparisons of Back Propagation Algorithm (BPA) network and Radial Basis Function (RBF) network for power quality disturbance classification. Features are extracted from the electrical signals by using Hilbert Huang Transform.

\section{Artificial Intelligence Based Classification Methods}

Both conventional and artificial intelligence (AI) based classification methods are reported in literature. Artificial intelligence (AI) may be broadly defined as the automation of activities that are associated with human thinking, such as decision-making, problem-solving, learning, perception, and reasoning [47] for the resolution of complex problems. In the classification of electrical disturbances, all of the factors that make AI a powerful tool are present. We get information which is massive electrical signals are constantly being received and distorted; there is an important noise component so that a classification of the disturbances must be carried out [48]. The main intelligent tools for PQ classification include expert systems, fuzzy logic, and artificial neural networks.

\section{V.1 Neural Network Based Classification}

Neural network is a nonlinear, data driven self adaptive method and is a promising tool for classification, and have been successfully applied to a variety of real world classification tasks in industry, business and science [49]. Applications include classification of power quality disturbances [50]. The neural network recognizes a given pattern by experience which is acquired during the learning or training phase when a set of finite examples is presented to the network. This set of finite examples is called the training set, and it consists of input patterns (i.e. input vector) along with their label of classes (i.e. output). In this phase, neurons 
in the network adjust their weight vectors according to certain learning rules. After the training process is completed, the knowledge needed to recognize patterns is stored in the neurons weight vectors. The network is then presented to another set of finite examples, i.e. the testing data set, to assess how well the network performs the recognition tasks. This process is known as testing or generalization. Artificial Neural Network (ANN) is a universal function approximator i.e. this can approximate any function with arbitrary accuracy. All the above mentioned attributes make ANN flexible in modeling real world complex problems [51]

The probabilistic Neural Network (PNN) is a supervised neural network that is widely used in the area of pattern recognition. The fact that PPNs offer a way to interpret the network's structure in terms of probability density functions (PDF) is an important merit of this type of networks in the learning processes [52]. The standard training procedure for PNNs requires a single pass over all to the patterns of the training set. This characteristic renders PNNs faster to train suitable for classification of power system faults. The architecture of PNN is composed radial basis layer and competitive layer. The PNN is a supervised neural network that is used for classification [53].

Santoso et al. [54] have classified six types of PQ events using wavelets and multiple neural networks. The classifier uses wavelet transform coefficient at five-scale signal decomposition level as input to multiple neural. The squared wavelet transform coefficients (SWTC) at each scale are used as inputs to the multiple neural networks for classifying the disturbances type. The architecture of the network is learning vector quantization (LVQ). The final decision for the disturbances type is made by combining the outcomes of multiple neural networks by using two decision making schemes. One is simple voting scheme and the other is Dempster-Shafer theory of evidence. Murat Uyar et al. [55] proposed an ST-based neural network classification process for the automatic classification of PQ disturbances. It performs a feature extraction and a classification algorithm composed of feature extractor based on time-frequency statistical features of the ST and a NN classifier based on multilayer perceptron (MLP) with RPROP learning algorithm. The algorithm is capable in classifying the distorted PQ signals accurately even under different noise conditions. Mishra et al. [53] proposed an S-transform based probabilistic neural network (PNN) classifier for classification of 11 types PQ disturbances with only four extracted features. Integrating S-transform with PNN can effectively detect and classify PQ disturbances even under noisy condition. Comparison of PNN with other two well known neural networks i.e., feed forward multilayer back propagation (FFML-BP) and learning vector quantization (LVQ) shows that PNN classifies events more effectively than FFML and LVQ.

\section{V.2 Support Vector Machine Based Classification}

Support Vector Machine (SVM) can be treated as a special neural network. In fact, a SVM model is equivalent to a two-layer, perceptron neural network. With using a kernel function, SVM is an alternative training method for multi-layer perceptron classifiers in which the weights of the network are identified by solving a quadratic programming problem under linear constraints, rather than by solving a non-convex unconstrained minimization as in standard neural network training [56]. SVMs are based on minimization of the misclassification probability of unseen patterns with an unknown probability distribution of data and have solid theoretical foundation rooted in statistical learning theory. Real world problems often require hypothesis spaces that are more complex than those using linear discriminants. SVMs are able to find non-linear boundaries if classes are linearly non-separable. The main issue of interest in using SVM for classification is its generalization performance. SVM performs better than neural networks in terms of generalization [57].

Axelberg et al. [58] proposed SVM based algorithm for classification of common types of voltage sag disturbances. The results have shown high classification accuracy which implies that, the SVM classification technique is an attractive choice for classification of voltage sag and other PQ disturbances. It has also been found that the accuracy of the proposed method is also dependent on the features given to the classifier. WheiMin Lin et al. [59] presented an integrated model for recognizing power quality disturbances using a novel wavelet multiclass support vector machine (WMSVM). Disturbance Events Detection System (DEDS) with WMSVMs was developed. WMSVMs were designed with simple network architecture to shorten the processing time. The proposed architecture could effectively detect information from distorted waves using WT and MSVM techniques. Valdomiro et al. [60] proposed a PQ disturbance detection and identification technique which combines advantages of disturbances identification strategy based on DWT, with the advantages of the ANNs and SVM to classify information automatically was implemented. Once the disturbance is detected, it is possible to locate it from the detail sequence at first decomposition level. Karthikeyan et al. [61] presented a wavelet transform and support vector machine based algorithm for classification of power quality disturbances. The features extracted through the wavelet transform are trained by a SVM for classification of power quality disturbances. Five types of disturbances are considered for the classification problem. The proposed approach using wavelet transform and support vector machine produces over all classification rate of $98.8 \%$. 


\section{V.3 Rule-based Expert Systems Classification}

Expert systems were proposed to identify, classify and diagnose power-system events successfully for a limited number of events [54]. An expert system for classification and analysis of voltage dips using Kalman filter for estimation of the amplitude has been shown in [62]. Rule-based expert systems are highly dependent on "if...then" clauses. To formulate a rule-based system for PQ disturbance classification, a knowledge base is composed using a set of rules in the form of expertise knowledge from a detailed analysis of the extracted features [63].

Chung et al. [64] presented a rule -based method used to classify time-characterized disturbances, and then, a wavelet method has been utilized to obtain a more flexible time frequency information. A hidden Markov model has also been adopted to determine the disturbance existence. Alex Wenda et al. [63] proposed a new approach for the automatic detection and classification of power quality disturbances through the Internet by combining the S-transform, a rule based expert system and a MATLAB web server. The S-transform is used to obtain the time frequency characteristics of power quality events under noisy conditions, and a set of features is extracted for pattern classification of power quality disturbances. A rule-based expert system is also developed in which the system classifies various power quality disturbances. Finally, a MATLAB web server is used to integrate the graphical and computational process with remote access through the internet.

\section{V.4 Fuzzy Expert System Based Classification}

It is usually appropriate to use fuzzy logic when a mathematical model of a process doesn't exist or does exist but is too difficult to encode and too complex to be evaluated fast enough for real time operation. The accuracy of the fuzzy logic systems is based on the knowledge of human experts; hence, it is only as good as the validity of the rules. As the power system data is highly uncertain and the power disturbance monitoring is a pattern classification problem [65].

Ortiz et al. [66] have proposed a fuzzy expert system for detection and classification of voltage sags. Abdelsalam et al. [67] proposed a new algorithm for power system disturbance classification. It is a two stage system that employs the great potentials of the discrete wavelet transform, Kalman filter and a fuzzy-expert system. For the first stage, the captured voltage waveform is passed through the DWT to determine the noise inside it. The covariance of this noise is then calculated and fed together with the captured voltage waveform to the Kalman filter to provide the amplitude and the slope of this waveform. These are considered as an input to the fuzzy-expert system in the second stage to determine the class to which the waveform belongs. Dash et al. [68] proposed a hybrid scheme using Fourier linear combiner and fuzzy expert system. The captured waveforms have been passed through a Fourier linear combiner block to extract amplitude and phase of the fundamental signal. The proposed method was found to be accurate and robust in presence of noise. It is computationally simple and gives classification result in less than a cycle.

\section{V.5 Genetic Algorithm Based Classification}

Genetic Algorithm (GA) is a probabilistic search method inspired by the biological evolution process [69]. The principle of GA is the survival of the fittest solutions among a population of potential solutions for a given problem. Thus, new generations produced by the surviving solutions are expected to provide better approximations to the optimum solution. The solutions correspond to chromosomes that are encoded with an appropriate alphabet. The fitness value of each chromosome is determined by a fitness function. New generations are obtained using genetic operators, crossover and mutation, with certain probabilities on the fittest members of the population. Initial population can be randomly or manually defined. Population size, number of generations, probability of crossover and mutation are defined empirically. The fitness value corresponding to a chromosome is usually defined as the classification accuracy obtained with the selected features [70].

Upender et al. [71] proposed a technique consisting of a preprocessing unit based on discrete wavelet transform in combination with genetic algorithm for classifying the power system fault disturbances. The DWT acts as extractor of distinctive features in the input current signals, which are collected at source end. The information is then fed into GA for classifying the faults. Brahmadesam et al. [72] proposed an efficient Genetic-Wrapper Algorithm based data mining for feature subset selection in a power quality pattern recognition application. The wrapper based approach integrates multi-objective genetic algorithms and the target learning algorithm in order to evolve optimal subsets of discriminatory features for pattern classification. The wavelet transform and the S-transform are utilized to produce representative feature vectors that can accurately capture the unique and salient characteristics of each disturbance.

\section{V.6 Adaptive Neuro-Fuzzy System Based Classification}

Adaptive Neuro-fuzzy system (ANFS) is a hybrid system incorporating the learning abilities of ANN and excellent knowledge representation and inference capabilities of fuzzy logic that have the ability to self modify their membership function to achieve a desired performance. An adaptive network, which subsumes 
almost all kinds of neural network paradigms, can be adopted to interpret the fuzzy inference system. ANFS utilizes the hybrid-learning rule and manage complex decision making or diagnosis systems. ANFS has proven to be an effective tool for tuning the membership functions of fuzzy inference systems [73].

An adaptive neuro fuzzy system to learn power quality signature waveform is proposed in [74]. The adaptive fuzzy systems are very successful in learning power quality waveform. The new adaptive neuro-fuzzy tool will enhance the performance of the existing power quality service. Chandra Sekhar et al. [75] proposed a hybrid approach of neuro-fuzzy based learning and classification approach based on the online learning systems. The effect of fault diagnosis for the suggested fault location tool is evaluated over the conventional fault diagnosis based approaches.

\section{Conclusion}

The problem of power quality has been discussed in this paper. This paper is a survey of work published on power quality disturbances and techniques for detection and classification of electrical power disturbance. The transformed feature extraction techniques and artificial intelligence techniques of PQ events classification are highlighted in particular. These methods are suitable for large and complex networks. This paper provides a general literature survey useful for the research on power quality disturbances in electrical power system.

\section{References}

[1] P.Daponte, M.di Penta, and G. Mercurio, Transientmeter: A distuributed measurement system for power quality monitoring, Proceeding $9^{\text {th }}$ International Conference Harmonics and Quality of Power, 2000, Vo. 3, 1017-1022.

[2] IEEE Standards Board, IEEE Std. 1159-1995, IEEE Recommended Practice for Monitoring Electric Power Quality (New York: IEEE, Inc. June, 1995).

[3] He H., Shen X., Starzyk J.A., Power quality disturbance analysis based on EDMRA method, Electrical Power and Energy System, Vol. 31, 2009, 258-268.

[4] R. Dugan, M. McGranaghan, and H. Wave Beaty, Electrical Power Systems Quality (N.Y.,McGraw-Hill, 1996).

[5] M.H.J. Bollen, Understanding Power Quality Problems-Voltage Sags and Interruptions (New York, IEEE Press, 2001).

[6] Inigo Monedero, Carlos Leon, Jorge Ropero, Antonio Garcia, Jose Manuel and Juan C. Montano, Classification of Electrical Disturbances in Real Time Using Neural Networks, IEEE Transaction on Power Delivery, 2007, 1-9.

[7] D. Saxena, K.S. verma and S.N. Singh, Power quality event classification: an overview and key issues, International Journal of Engineering, Science and Technology, Vol. 2, No.3, 2010, 186-199.

[8] S.Edwin Jose, and S.Titus, Detection and classification of Power Quality using Adaptive decomposition structure and neural network, European Journal of Scientific Research, Vol.89, No.3, October 2012, 477-489.

[9] R. swarna Latha, Ch. Sai Babu, and K. Durga Syam Prasad, Detection \& analysis of power quality disturbances using wavelet transforms and SVM, International research Journal of Signal Processing, Vol. 2, No. 2, 2001, 58-69.

[10] Emmanoiuil Styvaktakis, Irene Y.H. Gu, Math H.J. Bollen, Voltage dip detection and power system transients, IEEE Transactions Power Engineering Society Summer Meeting, Vol. 1, 15-19 July 2001, 683-688.

[11] Karimi M., Mokhtari H., and Iravani M.R., Wavelet based on-line disturbance detection for power quality application, IEEE Trans. Power Delivery, Vol.15, No. 4, 2000, 1212-1220.

[12] Axelberg P.G.V., Gu I.Y., and Bollen M.H.J., Support Vector Machine for classification of voltage disturbances, IEEE Trans. Power Delivery, Vol. 22, No. 3, 2007, 1297-1303.

[13] P. Kailasapathi, and D.Sivakumar, Methods to analyze power quality disturbances, European Journal of Scientific Research, Vol.47 No.1, 2010, 06-016.

[14] Anton V. Shupletsov, Irine V., and Hom Chneko, Wavelet packet transform for power quality factors measurement, Proc. of $5^{\text {th }}$ International Serabian Workshop and Tutorial (EDM 2004), Erlagol, July 15, 2004, 145-146.

[15] Bhim Singh, and Kamal Al-Haddadf, A review of active filters for power quality improvement, IEEE Transactions on Industrial Electronics, Vol 46, No. 5, Oct 1999.

[16] J.A. Rosendo, and A. Gomez, Efficient moving window-DFT algorithms, IEEE Transaction on Circuits and Systems, II: Analog and Digital Signal Processing 45, 1998, 256-260.

[17] Huang S.J., Hsieh C.T., and Huang C.L., Application of morlet wavelets to supervise power system disturbances, IEEE Transactions on Power Delivery, Vol.14, No.1, Jan 1999, 235-243.

[18] Pinnegar C.R., and Mansinha L., Time-local spectral analysis for non-stationary time series: the s-transform for noisy signals, Fluctuation and Noise Letters, Vol. 3, No. 4, L357-364.

[19] Dash P.K., Panigrahi B.K., Sahoo P.K., and Panda G., Power quality disturbance data compression, detection and classification using integrated spline wavelet and S-transform, IEEE Transactions on Power Delivery, Vol.18, No.2, 2003, 595-600.

[20] Lee I.W.C., and Dash P.K., S-Transform based intelligent system for classification of power quality disturbance signals, IEEE Transactions on Industrial Electronics, Vol.50, No.4, 2006, 800-805.

[21] Allen R.L., and Mills D., Signal analysis: time, frequency, scale, and structure (New York, NY, Willey, 2004).

[22] Stockwell R.G., Mansinha L., and Lowe R.P., Localization of the complex spectrum: the S-transform, IEEE Transactions on Signal Processing, Vol. 44, No.4, 998-1001.

[23] Raj Naindoo, and Pragasen Pilla, A new method of voltage sag and swell detection, IEEE Transactions on Power Delivery, Vol.22, No.2, 2007, 1056-1063.

[24] Vetrivel A., Malmurugan N., and Jerome J., A Novel Method of Power Quality Orthogonal S-transform (DOST) with wavelet support vector machine (WSVM) classifier, International Journal of Electrical Power Engineering, Vol. 3, No. 1, 59-68.

[25] Zbigniew Leonowicz, Tadeusz Lobos, and Krzysztof Wozniak, Analysis of non-stationary electric signals using the S-transform, The International Journal for Computation and Mathematics in Electrical and Electronics Engineering, Vol.28, No.1, 2009, 204210 .

[26] Ming He Zhang, Chen Xiaoqing, and Zhang Bin, Wavelet entropy measure definition and its application for transmission line fault detection, Proc. International Conference on Power System Technology, 2006, 1-5. 
[27] Ameen M. Gargoom, Nesmi Ertugrul, and Wen L.Soong, Automatic classification and characterization of power quality events, IEEE Transaction on Power Delivery, Vol.23, No.4, 1995, 2417-2425.

[28] Thai Nguyen, and Yuan Liao, Power quality disturbance classification utilizing S-transform and binary feature matrix method, Elsevier Journal Electric Power System Research, 79, 2009, 569-575.

[29] R. Yokeeswaran, and A. Vetrivel, Measurement and comparison of power quality disturbances using discrete wavelet transform (DWT) and discrete orthogonal S-transform, International Journal of Engineering Research and Application, Vol. 2, No. 3, MayJun 2012, 808-813.

[30] Subhamita Roy, and Sudipta Nath, Classification of power quality disturbances using features of signals, International Journal of Scientific and Research Publications, Vol. 2, No. 11, November 2012,01-09.

[31] Oshea P., A high resolution spectral analysis algorithm for power system disturbance monitoring, IEEE Transaction on Power System, Vol. 17, No. 3, 2002, 676-680.

[32] I. Daubechies, Ten lectures on wavelets, Philadelphia, PA:Society for Industrial and Applied Mathematics, (1992).

[33] C.S. Burrus, R.A. gopinath, and H.Guo, Introduction to Wavelets and Wavelet Transforms: A Primer (Englewood Cliffs, NJ: Prentice-Hall, 1998).

[34] Lalit Kumar Behera, Maya Nayak, and Sareeta Mohanty, Discrete wavelet transform and S-transform based time series data mining using multilayer perception neural network, International Journal of Engineering Science and Technology, Vol. 3 No. 11, November 2011, 8039-8046.

[35] Zhou Wenhui, Wavelet transform based new methods for voltage flicker signal and harmonic detection, The fifth International Conference on Power Electronics and Drive Systems, Vol.1, No. 17-20, 2003, 805-810.

[36] Daubuchies I., The wavelet transform, time frequency localization and signal analysis, IEEE transaction Information Theory, Vol. 36, No. 1, 2000, 184-190.

[37] Perumal Chandrasekar, and Vijayarajan Kamraj, Detection and classification of power quality disturbance waveform using MRA based modified wavelet transform and neural networks, Journal of Electrical Engineering, Vol. 61, No. 4, 2010, 235-240.

[38] P.F. Ribeio, Wavelet transform: an advanced tool for analyzing non-stationary harmonic distortions in power systems, Proceeding of the IEEE International Conference on Harmonics in Power Systems, Bologna, Itlay, September 1994.

[39] Zhenghu Li, Hilbert-Huang Transform based application in power system fault detection, International Workshop on Intelligent Systems and Applications, ISA 2009, 01-04.

[40] N.E. Huang, Z.Shen, S.R. long, M.C. Wu, H.H. Shih, Q. Zheng, N.C. Yen, C.C. Tung, and H.H.Liu, The empirical mode decomposition and the Hilbert spectrum for nonlinear and non-stationary time series analysis, Proc. R.Soc. Lond. A, Vol. 454, 1998, 903-995.

[41] J.C. Echeverria, J. Crowe, M. Woolfson, and B.R. Hayes-Gill, Application of empirical mode decomposition to heart rate variability analysis, Med. Biol. Eng. Comput., Vol. 39, No. 4, 2001, 471-479.

[42] B.M. Battista, C. Knapp, T. McGee, and V.Goebel, Application of the empirical mode decomposition and Hilbert Huang transform to seismic reflection data, Geophysics, Vol.72, No. 2, 2007, H29-H37.

[43] M.A. Andrade, A.R. Messina, C.A. Rivera, and D.Olguin, Identification of instantaneous attributes of torsional shaft signals using the Hilbert transform, IEEE Transaction on Power System, Vol. 19, 2004, 1422-1429.

[44] T. Jayasree, D. Sam Harrison, and T. Sree Rangaraja, Automated classification of power quality disturbances using Hilbert Huang Transform and RBF networks, International Journal of Soft Computing and Engineering, Vol. 1, No. 5, November 2011, 217-222.

[45] Nilanjan Senroy, Siddharth Suryanarayanan, and Paulo F. Ribeiro, An improved Hilbert-Huang method for analysis of time-varying waveforms in power quality, IEEE Transactions on Power Systems, Vol. 22, No. 4, November 2007, 1843-1850.

[46] M.K. Elango, A. Nirmal Kumar, S. Purushothaman, Application of neural networks for power quality disturbance classification using Hilbert Huang transform, European Journal of Scientific Research, Vo. 47, No. 3, 2010, 442-454.

[47] W.R. Anis Ibrahim, and M.M. Morcos, Artificial intelligence and advanced mathematical tools for power quality applications: A survey, IEEE Transactions on Power Delivery, Vol. 17, No.2, April 2002, 668-673.

[48] W.E. Kazibwe, and H.M. Sendaula, Expert Systems Targets. Power Quality Issues(New York, IEEE, April 1992).

[49] Widrow B., Rumelhard D.E., and Lehr M.A., Neural networks: Applications in industry, business and science, Communication ACM, Vol. 37, 1994, 93-105.

[50] M. Manjula, and AVRS Sarma, Assessment of power quality events by empirical mode decomposition based neural network, Proceeding of the World Congress on Engineering 2012,London U.K., WCE 2012, July 4-6,Vol. II.

[51] Zhang G.P., Neural Networks for classification: A survey, IEEE Transactions on Systems, Man, and Cybernetics-Part C: Applications and Reviews, Vol. 30, No. 4, November 2000, 451-462.

[52] Specht D.F., Probabilistic neural networks, Neural Network, Vol. 3, 1990, 109-118.

[53] Mishra S., Bhende C.N., and Panigrahi B.K., Detection and classification of power quality disturbances using S-transform and probabilistic neural network, IEEE Transactions on Power Delivery, January 2008, Vol. 23.

[54] Santoso S., Lamoree J., Grady W.M., Powers E.J., and Bhatt S.C., A scalable PQ event identification system, IEEE Transactions on Power Delivery, Vol.15, 2000, 738-743.

[55] Murat Uyar, Secuk Yildirim, and Muhsin Tunay Gencoglu, An expert system based on S-transform and neural network for automatic classification of power quality disturbances, International Journal of Expert Systems with Applications, Vol. 36, 2009, 5962-5975.

[56] Davood Dehestani, Fahimeh Eftekhari, Ying Guo, Steven Ling, Steven Su, and Hung Nguyen, Online support vector machine application for model based fault detection and isolation of HVAC system, International Journal of Machine Learning and Computing, Vol. 1, No. 1, April 2011.

[57] N. Cristianini, and J. Shawe Taylor, An Introduction to Support Vector Machines and other Kernel based Learning Methods (Cambridge, U.K., Cambridge University Press, 2000, 31-112).

[58] Axelberg P.G.V., Gu I.Y.H., and Bollen M.H.J., Support vector machine for classification of voltage disturbances, IEEE Transactions on Power Delivery, Vol. 22, No. 2, 1740-1742.

[59] Whei-Min Lin, Chien-Hsien Wu, Chia-Hung Lin, and Fu-Sheng Cheng, Detection and classification of multiple power quality disturbances with wavelet multiclass SVM, IEEE Transactions on Power Delivery, Vol. 23, No.24, October 2008, 2575-2582.

[60] Valdomiro Vega, Cesar Duarte, and Gabriel Ordonez, Automatic power quality disturbances detection and classification based on discrete wavelet transform and support vector machines, CIRED $19^{\text {th }}$ International Conference on Electricity Distribution, Vienna, 21-24 May 2007, paper No. 0827, 01-04.

[61] M. Karthikeyan, and V.Malathi, Wavelet-support vector machine approach for classification of power quality disturbances, International Journal of Recent Trends in Engineering, Vol. 1, N0. 3, May 2009, 290-293. 
[62] E. Styvaktakis, M.H.J. Bollen, and I.Y.H. Gu, Expert system for voltage dip classification and analysis, IEEE Power Engineering Society Summer meeting, Vol. 1, July 2001, 671-676.

[63] Alex Wenda, Aini Hussain, M.A. Hannan, Azah Mohamed, and Salina Abdul Samad, Web based automatic classification of power quality disturbances using the S-tranform and a rule based expert system, Journal of Information \& Computational Science, Vol. 8, No. $12,2001,2375-2383$.

[64] Chung J., powers E.J., Grady W.M., and Bhatt S.C., Power disturbance classifier using a rule-based method and wavelet packetbased hidden markov model, IEEE Transactions on Power delivery, Vol. 17, 233-241.

[65] Mendel Jerry M., Fuzzy logic systems for engineering; a tutorial, IEEE Proc., Vol. 83, No. 3, 1995, 345-377.

[66] Ortiz A., Manana M., Ortiz F., Delgado F., and Eguiluz L.I., Implementation of a voltage sag characterization based on DSP platform, Proceedings of ICREPQ-06, Palma de Mallorca, 2006.

[67] A.A. Abdelsalam, A.A. Eldesouky, and A.A. Sallam, Wavelet, Kalman Filter and Fuzzy-Expert combined system for classification power system disturbances, Proceedings of $14^{\text {th }}$ International Middle East Power Systems Conference(MEPCON-10), Cairo University, Egypt, December 19-21, 2010, 398-403.

[68] Dash P.K., Mishra S., Salama M.M.A., and Liew A.C., Classification of power system disturbances using a fuzzy expert system and a Fourier linear combiner, IEEE Transactions on Power Delivery, Vol. 15, 2000, 472-477.

[69] Goldberg D.E., Genetic algorithms in search, optimization, and machine learning (Reading, MA: Addison-Wesley, 1989).

[70] Yang J., Honavar V., Features subset selection using a genetic algorithm, IEEE Intelligent Systems, Vol. 13, No. 2, 1998, 44-49.

[71] J.Upendar, C.P. Gupta, and G.K. Singh, Discrete wavelet transform and genetic algorithm based fault classification of transmi ssion systems, $15^{\text {th }}$ National Power Systems Conference, IIT Bombay, December 2008, 323-328.

[72] Brahmadesam Krishna, and Baskaran Kaliaperumal, Efficient Genetic-wrapper algorithm based data mining for feature subset selection in a power quality pattern recognition application, The International Arab of Information Technology, Vol. 8, no. 4, October 2011, 397-405.

[73] Lin C.T., and Lee C.S.G., Neural network based fuzzy logic control and decision system, IEEE Transaction on Computers, Vol. 40, No.12, 1991, 1320-1336.

[74] Ibrahim W.R.A., and Morcos M.M., An adaptive fuzzy technique for learning power quality signature waveforms, IEEE Power Engineering Review, Vol. 21, No. 1, 2001, 56-58.

[75] P. Chandra Sekhar, B.V. Sanker Ram, and K.S. Sarma, Neuro-Fuzzy approach for fault location and diagnosis using online learning system, Journal of Theoretical and Applied Information Technology, 48-55. 